Introduction Chronic nonbacterial osteomyelitis is a rare autoinflammatory disease of unknown etiology that primarily affects bone and presents with sterile inflammation of the most common metaphyseal areas of the long bones, clavicle, spine, and pelvis. Affected bones are prone to pathologic fractures and slow growth. Laboratory workup results are nonspecific, and skeletal scintigraphy with technetium and magnetic resonance imaging are used as diagnostic imaging modalities. Differentially, malignant events must be considered, such as leukemia and malignant bone tumors, chronic infectious osteomyelitis, avascular necrosis, etc. If the diagnosis is unclear, a bone biopsy with bacteriological analysis of the bone sample is required to exclude infectious osteomyelitis.

Case Report An 8-year-old girl was treated for acute lymphoblastic leukemia of $\mathrm{T}$ immunophenotype, high risk, according to ALL IC-BFM 2009 protocol, early complete remission was achieved. Intensive chemotherapy treatment was characterized by a number of complications, including frequent febrile neutropenia, invasive fungal lung disease, and acute neurotoxicity in the form of cerebral ischemia. After completion of reinduction therapy, a period of persistent bone marrow aplasia followed by recurrent and prolonged sepsis caused by a highly resistant strain of Pseudomonas aeruginosa of unknown origin preceded maintenance therapy. Because the girl complained of foot pain with inability to walk during maintenance therapy, extensive diagnostic workup was performed, including technetium bone scintigraphy and magnetic resonance imaging, to verify an osteomyelitis focus in the left calcaneus with intense bone remodeling of SI and shoulder joint. After completion of treatment for sepsis in the control laboratory findings continued slightly elevated C-reactive protein with normal sedimentation of erythrocytes and procalcitonin. Pathohistological analysis of bone biopsy showed chronic inflammatory reaction, while microbiological analysis was negative. Skin lesions were not observed, HLA typing was negative, anamnestic data of maternal inflammatory bowel disease was known. In collaboration with an immunoreumatologist and an orthopedist, chronic nonbacterial osteomyelitis was treated and treatment with bisphosphonates was started. according to a standard regimen, with an excellent clinical response. The girl was pain free six months after starting treatment and physical therapy. The underlying malignancy is still in remission, inflammatory parameters are normal, while control imaging still continues.

Conclusion Chronic nonbacterial osteomyelitis should be considered as a differential diagnosis in chronic inflammatory bone lesions without an isolated microbiologic agent and in patients with acute lymphocytic leukemia. Therapeutic options include nonsteroidal anti-inflammatory drugs, disease-modifying antirheumatic drugs (DMARDs), corticosteroids, and bisphosphonates. However, given the possibility of influencing hematologic findings, bisphosphonate therapy is the treatment of choice if oncologic disease requires further treatment.

\section{TREATMENT RESULTS OF T-LYMPHOBLASTIC LYMPHOMA IN CHILDREN - A SINGLE-CENTER EXPERIENCE}

\footnotetext{
${ }^{1,2}$ Ernest Bilić, ${ }^{2}$ Petra Ivančićc, ${ }^{1}$ Maja Pavlović, ${ }^{1}$ Ranka Femenić, ${ }^{1}$ Ana Petrović-Gluščić,

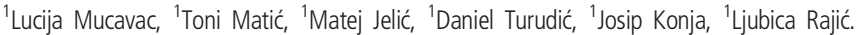
${ }^{1}$ Department of Pediatrics, Division of Hematology and Oncology, University Hospital Centre Zagreb, Zagreb, Croatia; ${ }^{2}$ School of Medicine, University of Zagreb, Zagreb, Croatia
}

10.1136/archdischild-2021-europaediatrics.323
Aim Our study aimed to determine the overall survival of children with non-Hodgkin T-lymphoblastic lymphoma (TLBL), analyze and present patient characteristics and initial presentation of their disease.

Methods Our study included all patients with T-LBL nonHodgkin lymphoma treated in the Department of Pediatrics, Division of Hematology and Oncology, University Hospital Centre Zagreb between January 1st, 2002 and December 31st, 2017. Relevant information including general patient data (gender, age at the time of diagnosis), initial clinical presentation (the presence of mediastinal masses, pericardial and pleural effusion, the presence of bone marrow and central nervous system disease) and treatment information (used therapeutic protocol and mortality data) was collected from the available medical documentation. The Kaplan-Meier curve shows patients' survival.

Results Overall, 19 patients were included, 14 of which were male $(73.68 \%)$ and 5 were female $(26.32 \%)$. The median age at diagnosis was 10 years. 17 patients (94.44\%) presented with mediastinal mass and 9 of them $(52.94 \%)$ had pleural effusion. At the time of diagnosis, pericardial effusion and superior vena cava syndrome were present in $6(33.33 \%)$ and $3(16.67 \%)$ patients, respectively. Bone marrow involvement was detected in 10 patients (52.63\%), whereas CNS involvement in only one (5.26\%). 16 out of 19 patients survived (84.21\%; 95\% CI 67.81-100). Survival was higher for boys $(85.71 \%)$ than for girls $(80 \%)$, but the difference was not statistically significant $(\mathrm{p}=0.764)$.

Conclusion Comparing general epidemiologic data with the one in the available literature we haven't found a significant deviation of the prevalence of the disease between the sex or the median age of onset of the disease. The result of $90 \%$ five-year survival of children with T-LBL in the BFM group study was not repeated in later studies and the overall survival of $84 \%$ in our group was consistent with the results reported in the available literature.

\section{NEUTROPENIA IN CHILDREN}

${ }^{1,2}{ }^{2}$ rnest Bilićx, ${ }^{2}$ Kristijan Lujić, ${ }^{1}$ Maja Pavlović, 'Lucija Mucavac, ${ }^{1} T o n i$ Matić, ${ }^{1}$ Daniel Turudić, ${ }^{1}$ Matej Jelić, ${ }^{2}$ Hana Chudy. ${ }^{1}$ University Hospital Centre Zagreb, Department of Pediatrics, Division of Pediatric Hematology and Oncology; ${ }^{2}$ School of Medicine, University of Zagreb, Zagreb, Croatia

\subsection{6/archdischild-2021-europaediatrics.324}

Aim The aim of this study was to show presentation of individual types of neutropenia as well as their outcome depending on the lowest absolute neutrophil count (ANC), period in which ANC was below 1000 and presence of infections during the duration of neutropenia.

Materials and Methods In this study 288 children with neutropenia were observed in the period from 2005-2020. The study was provided at the Division of Pediatric Hematology and Oncology at the University Hospital Centre Zagreb, Croatia.

Results $139(48,26 \%)$ examinees were female and 149 $(51,74 \%)$ were male. Of all the examinees 265 (92,01\%) were diagnosed before their seventh birthday while 175 (60,76\%) had diagnosis made during their first year of life. Retrospective analysis revealed that $281(97,57 \%)$ examinees had acquired neutropenia and only $7(2,51 \%)$ had some type of congenital neutropenia ( 3 had severe congenital neutropenia, 2 had cyclic neutropenia, 1 had Shwachman-Diamond syndrome 
and 1 had methylmalonic aciduria). Average duration of neutropenia was 6,43 months while average age at diagnosis was 19,6 months. During this study 227 (78,82\%) examinees were cured, $34(11,81 \%)$ were still being followed up and for 27 $(9,38 \%)$ of them we didn't know outcome. 39,24\% neutropenias were acute and $56,25 \%$ were chronic. According to the severity of neutropenia the distribution was $60,42 \%-26,39 \%$ - $12,85 \%$ (severe, moderate, mild). Average duration of neutropenia in cases of severe neutropenia (ANC $<0,5 \times 10^{9} / 1$ ) was 9,14 months, in moderate neutropenia (ANC $=0,5-1,0 \mathrm{x}$ $10^{9} / 1$ ) 4,09 months and in mild neutropenia (ANC > 1,0 $\mathrm{x}$ $\left.10^{9} / 1\right) 1,64$ months. We recorded infection during neutropenia in $232(80,56 \%)$ examinees while 88 (30,56\%) had noted infections before the onset of neutropenia.

Conclusion This study showed that $97,57 \%$ children had benign neutropenia and $78,82 \%$ were spontaneously cured during the research which are encouraging results. We noted that children with mild neutropenias and those who had no recorded infections during neutropenia had shorter average duration of neutropenia. In conclusion, most neutropenias of the early childhood are benign and have favorable outcome.

\section{APLASTIC CRISIS INDUCED BY HUMAN PARVOVIRUS B19 AS AN INITIAL PRESENTATION OF HEREDITARY SPHEROCYTOSIS IN A CHILD - A CASE REPORT}

Petra Ivančić* ${ }^{*}$ Maja Vrdoljak. Medicinski fakultet Sveučilišta u Zagrebu

\subsection{6/archdischild-2021-europaediatrics.325}

Background A transient, self-limiting aplastic crisis is a rare manifestation of parvovirus (PV) B19 infection, usually seen in patients with underlying haemolytic anaemias. The virus has a predilection for infecting the erythroid progenitor cells of the bone marrow resulting in their lysis and red cell aplasia, although white cell and platelet counts may also decline.

We herein report aplastic crisis induced by PV B19 infection unmasking hereditary spherocytosis in a boy.

Case Presentation A 11-year-old boy presented with high-grade fever, headache, drowsiness, sore throat, and a rash.

On admission he was conscious, but sleepy, drooling, with pale skin and conjunctivae. Physical examination revealed macular, somewhere petechial rash involving the neck and extremities, tachycardia, systolic murmur and hepatosplenomegaly.

Complete blood count revealed RBC of 2.34 x10e12/L, with haemoglobin concentration of $64 \mathrm{~g} / \mathrm{L}$ and reticulocytopenia, WBC $1.3 \times 10 \mathrm{e} 9 / \mathrm{L}$, and platelet count $75 \times 10 \mathrm{e} 9 / \mathrm{L}$. Spherocytosis was present on the peripheral blood smear.

Bone marrow aspirate showed suppressed erythropoiesis. The PV B19 infection was diagnosed by polymerase chain reaction (292 $000000 \mathrm{DNA}$ copies/ml of blood) and positive serology for specific anti-PV B19 IgM.

Osmotic fragility testing showed increased fragility of erythrocytes, which was consistent with the diagnosis of hereditary spherocytosis.

The boy was treated by blood and platelet transfusions and supportive care, and was discharged after 9 days of hospital care with improved blood count.

Conclusions PV B19 induced aplastic crisis can be the first manifestation of hereditary spherocytosis. PV B19 infection must be considered in the differential diagnosis in patients with acquired aplastic anaemia.

\section{AUTOSPLENECTOMY - CASE REPORT}

Dora Šercar*, Izabela Kranjěec, Nuša Matijašić. Department of Oncology and Hematology, Children's Hospital Zagreb, Zagreb, Croatia

\subsection{6/archdischild-2021-europaediatrics.326}

Introduction Autosplenectomy defines spontaneous splenic infarction leading to hyposplenism. It occurs mainly as a complication of sickle cell anemia, pneumococcal sepsis or systemic lupus erythematosus, but may also be associated with various other conditions. An anatomical variation of the spleen's position, the wandering spleen, has been reported as an underlying cause in less than $0.5 \%$ of autosplenectomy cases.

Case Report A 9.5-month-old female was admitted to the Department of Oncology and Hematology of the Children's Hospital Zagreb due to a prolonged fever of an unknown origin and splenomegaly. Laboratory findings detected profound normocytic anaemia, attributed to splenic sequestration. Abdominal ultrasound detected multiple hypoechoic areas in the spleen, indicating splenic infarction, confirmed by MR angiography. An extensive work-up excluded sickle cell anemia, thalassemia, hematological malignancies, myeloproliferative disorders, thrombophilia, autoimmune vasculitis, Kawasaki disease and infection as a cause of the condition. However, repeated ultrasound examination revealed an aberrant, ventrally positioned spleen, which most likely led to an inadequate blood supply and consequently splenic infarction. Antiplatelet therapy and antibiotic prophylaxis were initiated, along with pneumococcal and meningococcal vaccines. The patient is still monitored regularly, currently with normal hematological laboratory findings and none of the complications possibly attributed to hyposplenism.

Conclusion Autosplenectomy in children, although rarely, may result from an aberrant spleen's position. Congenital weakness of the splenic ligaments, allowing its mobility, causes torsion of the splenic artery, leading to blood supply failure and tissue damage. Given the wide differential diagnosis of splenic infarction, it is necessary to exclude numerous underlying medical conditions, primarily sickle cell anemia. A conservative approach is currently the treatment of choice. Hyposplenism, leading to susceptibility to sepsis caused by encapsulated bacteria, requires appropriate antibiotic prophylaxis and vaccination of such patients.

\section{Paediatric Intensive and Emergency Medicine}

\section{DIFFICULTIES EMERGING FROM THE END-OF-LIFE CARE IN THE PEDIATRIC INTENSIVE CARE UNITS}

Filip Rubic*, M Curkovic, S Jankovic, L Brajkovic, A Jozepovic, M Novak, B Filipovic-Grcic, A Borovecki. University Hospital Zagreb

10.1136/archdischild-2021-europaediatrics.327

Working in ICUs that involves care for critically ill children is inherently demanding. The intricacy of end-of-life issues in this setting adds additional layer of high demands that health care professionals are inadequately prepared for An interpretative, qualitative inquiry based on thematic data analysis using focus groups as data collection method was used in order to 